\title{
Association of Apolipoprotein $E$ Polymorphisms with White Matter Lesions and Brain Atrophy
}

\author{
ZhiLi Niu, PingAn Zhang ${ }^{\bowtie}$, Dong Li, ChengLiang Zhu, LiNa Feng, \\ Ge Xiong, NaNa Song, Pei Tang, and Feng Liu \\ Department of Laboratory Science, Renmin Hospital of WuHan University, Wuhan, China
}

\begin{abstract}
Objective Apolipoprotein E (ApoE) is mainly synthesized in the liver. So far, it is unknown the relationship among APOE gene polymorphisms and WML, brain atrophy. Therefore, the aim of the study was to assess the associations of APOE gene polymorphisms in patients with WML and brain atrophy.

Methods A total of 58 patients with WML, 128 patients with brain atrophy, 112 patients with co-occurrence of WML and brain atrophy and 95 healthy elderly volunteers were recruited from Renmin Hospital of WuHan University.

Results Allele E3 was the most common allele. The alleles E2 had significantly higher levels of ApoB and lower age in WML group. The alleles E2 was associated with the lower level of ApoB, LDL-Ch, TCh, and sdLDL in co-occurrence group. The E3/E3 genotype has higher level of sdLDL, but lower age and female frequency in WML. The E3/E4 genotype had higher level of TG, but lower age in WML. Gender, Age, E2, Hyperhomocysteinemia and UA were also significantly associated with disease progression.

Conclusion This study found that clinical data, lipids and metabolic complications were closely related to ApoE genotypes and alleles, and also disease progression and type.

Psychiatry Investig 2020;17(2):96-105
\end{abstract}

Key Words Apolipoprotein E, Polymorphisms, Brain atrophy, WML.

\section{INTRODUCTION}

Apolipoprotein E (ApoE) is a 34-kDa glycoprotein with 299 amino acids that is mainly synthesized in the liver. The apoE gene with $3.6 \mathrm{~kb}$ is located on chromosome 19q13.32. ${ }^{1}$ APOE has three major isoforms, $A P O E 2, E 3$, and $E 4$, which are encoded by three alleles (E2,E3, and $E 4)$, resulting in six different genotypes (E2/E2, E3/E3, E2/E3, E3/E4, E2/E4, and E4/ $E 4) .{ }^{2,3}$ The $E 3$ allele differs from the $E 2$ allele by change cysteine to arginine at codon 158, while the $E 4$ differs from $E 3$ by a substitution of arginine for cysteine at residue 112. ApoE protein plays an important role in lipoprotein metabolism. E2 allele was associated with decreased plasma cholesterol level but E4 with elevations in LDL-C. Moreover, some studies have re-

Received: April 26, 2019 Revised: September 27, 2019

Accepted: October 28, 2019

$\triangle$ Correspondence: PingAn Zhang, MD, PhD

Department of Laboratory Science, Renmin Hospital of WuHan University, Wuhan City, Hu Bei Province 430060, China

Tel: +86-027-88041911-83456, Fax: +86-027-88071553

E-mail: zhangpingan927@163.com

(c) This is an Open Access article distributed under the terms of the Creative Commons Attribution Non-Commercial License (https://creativecommons.org/licenses/bync/4.0) which permits unrestricted non-commercial use, distribution, and reproduction in any medium, provided the original work is properly cited. ported that the E4 allele is associated with the risk of coronary heart disease. ${ }^{4}$

Brain atrophy was mainly caused by cell apoptosis and considered as an irresistible physiological process, characterized by widening of the sulci, decreased specific grey or white matter volumes and increased ventricular volumes, which was a common finding on MRI in the elderly. ${ }^{5,6}$ White matter disease (WMD) was a neuroimaging term, also known as leukoaraiosis (LA). The concept of LA was first proposed by Hachinski in $1987 .{ }^{7}$ WML appeared as a speckled or patchy change in the subcorolla or periventricular white matter, showing a low-density lesion on CT, and also T1WI image with low signal or an equal signal, and the T2WI and T2 FLAIR images with high signals on the MRI. In addition to brain atrophy, WML was considered as the result of cerebral small-vessel disease and also often found on MRI in the elderly. ${ }^{8,9}$ Brain atrophy and WML had many of the same features as followed. First, evidence has accumulated that vascular factors including hypertension, hyperlipidemia, diabetes mellitus, obesity, alcohol, and smoking play an important role in the etiology of brain atrophy and WML. ${ }^{10}$ Second, they were associated with future cognitive decline and dementia. ${ }^{11,12}$ Then, they were common 
accompaniment of ageing and accelerate in older age.

Several studies had demonstrated the association of APOE polymorphisms with cardiovascular and nervous system diseases, Alzheimer's disease, diabetic nephropathy, atherosclerosis, diabetes. ${ }^{13,14}$ Few studies had investigated apoE polymorphism in the Chinese population with White matter lesions and/or brain atrophy. More recently, concomitant brain atrophy and WML have frequently been observed in elderly people on magnetic resonance imaging (MRI). However, it is unknown which factors may explain the co-occurrence of WML and brain atrophy. Therefore, the objective of this study was to assess the genetic associations of APOE allele/genotype frequencies in patients with White matter lesions and/or brain atrophy.

\section{METHODS}

\section{Participants}

Patients and controls were recruited from Renmin Hospital of WuHan University, Hubei Province in China from January 2018 to December 2018. This study included 393 participants, which consisted of 58 patients with White matter lesions, 128 patients with White matter lesions, 112 patients with White matter lesions and 95 healthy elderly volunteers who underwent a regular health examination as healthy controls. WMLs was identified on MRI scan and its severity was graded using the Fazekas method to include a score for the deep white matter and periventricular regions when patients were rechecked MRI scan. ${ }^{15}$ The brain atrophy disease was scored on the frontal, temporal, and parietal atrophy by the cortical atrophy scale and the subject's head MRI T1 image. ${ }^{16}$ The above assessment was performed by two neurologists who were blinded to the basic information of the patients. Written informed consent was obtained from all participants before the collection of biological samples, and the protocol was approved by the Medical Ethics Review Committee of Renmin Hospital of WuHan University (WDRY2019-K105).

\section{Biochemical measurement}

Approximately $5 \mathrm{~mL}$ of blood was taken from each study participant in the morning after 12-hour overnight fast and all subjects were told to consume a bland diet before blood testing, and serum samples were separated immediately by centrifugation. Serum samples were stored at $-80^{\circ} \mathrm{C}$ until analysis. Serum high density lipoprotein cholesterol (HDL-C), low density lipoprotein cholesterol (LDL-C), total cholesterol (TCh), triglyceride (TG), small dense density lipoprotein (sdLDL), uric acid (UA), creatinine (Cr) were measured by enzymatic methods with commercially available kits on SEIMENS ADVIA 2400 (Siemens Healthcare Diagnostics ltd, Frimley, Camberley, UK). Lipoprotein(a) [Lp(a)], apolipoprotein A1 (Apo-
A1), and apolipoprotein B (Apo-B) levels were measured by immunoturbidimetric method, using reagent from SEIMENS ADVIA 2400 (Siemens Healthcare Diagnostics Inc, Akishima, Tokyo, Japan). Reference intervals were: ApoA1: 1.0-1.6 g/L, ApoB: 0.75-1.00 g/L, HDL-Ch: 1.00-1.55 mmol/L, LDLCh: 1.9-3.1 mmol/L, Lp(a): <300 mg/L, TCh: 3.1-5.2 mmol/L, TG: 0.6-1.7 mmol/L, sdLDL: 0.25-1.17 mmol/L, UA: 155-357 $\mu \mathrm{mol} / \mathrm{L}$, Urea: $2.6-7.5 \mathrm{mmol} / \mathrm{L}, \mathrm{Cr}: 41-73 \mu \mathrm{mol} / \mathrm{L}$. In addition to Biochemical Measurement, the following risk factors were also recorded for each individual: Hyperuricemia, Hypertension, Hyperhomocysteinemia, Hyperlipidemia, Type 2 diabetes.

\section{DNA extraction and APOE genotyping}

Genomic DNA was extracted from peripheral blood mononuclear cells by using a QIAamp DNA Blood Mini Kit [Kaijie Enterprise Management (Shanghai) Co., Ltd., Xuhui, Shanghai, China] following the manufacturer's instructions, and DNA concentration was quantified by using a NanoDrop $2000^{\mathrm{TM}}$ spectrophotometer (ThermoFisher Scientific, Waltham, MA, USA). PCR was performed according to the following protocol: $50^{\circ} \mathrm{C}$ for two minutes, pre-denaturation at $95^{\circ} \mathrm{C}$ for $15 \mathrm{~min}-$ utes, followed by 45 cycles at $94^{\circ} \mathrm{C}$ for 30 seconds and $65^{\circ} \mathrm{C}$ for 45 seconds. The amplified products were detected by using an APOE Gene typing Detection kit (gene-chip assay) (Sinochips Bioscience Co., Ltd., Zhuhai, Guangdong, China) (@ Association of APOE Gene Polymorphisms with Cerebral Infarction in the Chinese Population).

\section{Genetic analysis}

The detection of three isoforms of the apoE protein (E2, E3, and E4) resulting from the polymorphisms of the gene was performed as described in the literature. ${ }^{17}$

\section{Statistical analysis}

Statistical Package for the Social Sciences (SPSS) version 19.0 (IBM Corp., Armonk, NY, USA) was used for data analysis. The data were reported as mean \pm standard deviation (SD), quarter median and frequencies. The chi-square test and ANOVA were used to analyze the association between specific APOE genotypes and clinical characteristics. Multivariate logistic regression analysis was carried out to estimate the odds ratio (OR), with 95\% confidence intervals (CI), in order to assess basic information, clinical indicators and APOE genotypes and alleles risk factors for progress of the disease. The results were considered to be significant differences when the $\mathrm{p}$ value was less than 0.05 . 


\section{RESULTS}

\section{Clinical characteristics of the patient and control groups}

As showed in Table 1, comparison between disease group (White matter lesions, brain atrophy, Combined with white matter lesions and brain atrophy) and the controls indicated statistically significant differences in the clinical parameters, which included Gender $(\mathrm{p}<0.0001)$, Age $(\mathrm{p}<0.0001)$, ApoB $(\mathrm{p}<0.0001)$, HDL-Ch $(\mathrm{p}=0.005), \operatorname{Lp}(\mathrm{a})(\mathrm{p}=0.001), \mathrm{TCh}(\mathrm{p}<$ $0.0001)$, TG $(\mathrm{p}<0.0001)$, sdLDL $(\mathrm{p}<0.0001)$, UA $(\mathrm{p}<0.0001)$, Urea $(\mathrm{p}=0.002), \mathrm{Cr}(\mathrm{p}<0.0001)$, except for LDL-Ch and ApoA1.

\section{The distribution and frequencies of the APOE genotypes and alleles in the patient and control groups}

Table 2 showed that carriers of genotype E3/E3 was the most common type in three groups $(65.52 \%, 69.53 \%, 57.14 \%$ of White matter lesions, brain atrophy, and Combined with brain atrophy and white matter lesions groups, respectively), followed by E3/E4 genotype (22.41\%, 17.97\% of White matter lesions and brain atrophy groups, respectively), but the genotype frequency of $E 2 / E 3$ was higher than $E 3 / 4$ in Combined with white matter lesions and brain atrophy group $(\mathrm{p}=0.016)$. Allele $E 3$ was the most common allele, followed by allele $E 4$ and allele $E 2$ in White matter lesions, brain atrophy, and Combined with white matter lesions and brain atrophy groups, re- spectively ( $\mathrm{p}=0.011$ ). The type 2 diabetes of combined metabolic disease was significantly different among the three groups $(\mathrm{p}=0.015)$. However, others including Hyperuricemia, Hypertension, Hyperhomocysteinemia and Hyperlipidemia were not significantly different.

\section{Comparison of metabolic parameters among the different ApoE alleles}

We further analyzed the effects of the APOE genotypes on the clinical and metabolic parameters in White matter lesions group. As shown in Table 3, Age and the levels of ApoB and Urea were significantly different among the APOE genotype groups in White matter lesions group. Specifically, the subjects with an alleles $E 4$ was associated with the lowest level of Urea incidence ( $\mathrm{p}=0.021$ ), however, subjects with the alleles $E 2$ had significantly higher levels of $\mathrm{ApoB}$ and lower age compared with the $E 3$ and $E 4$ group ( $\mathrm{p}=0.040, \mathrm{p}=0.026$, respectively). In addition, we compared the relationship between $A p o E$ alleles and Combined metabolic disease, which was not statistically significant $(\mathrm{p}>0.05)$

\section{Comparison of metabolic parameters among the different ApoE alleles}

We also analyzed the effects of the APOE genotypes on the clinical and metabolic parameters in brain atrophy group. As shown in Table 4 , there was no significantly different among clinical, metabolic parameters and $A P O E$ genotype groups in

Table 1. Comparison of clinical data between disease group and control group

\begin{tabular}{|c|c|c|c|c|c|}
\hline Clinical characteristics & WML $(\mathrm{N}=58)$ & Brain atrophy $(\mathrm{N}=128)$ & $\begin{array}{l}\text { Co-occurrence of WML } \\
\text { and brain atrophy }(\mathrm{N}=112)\end{array}$ & $\begin{array}{l}\text { Control group } \\
(\mathrm{N}=95)\end{array}$ & $\mathrm{p}$ value \\
\hline Gender (male, \%) & $29(50.00)$ & $92(71.88)$ & $73(65.18)$ & $38(40.00)$ & $<0.0001$ \\
\hline Age (years) & $62.9 \pm 10.06$ & $70.58 \pm 9.54$ & $71.58 \pm 8.89$ & $64.12 \pm 10.66$ & $<0.0001$ \\
\hline ApoA1 (g/L) & $1.35 \pm 0.222$ & $1.31 \pm 0.206$ & $1.30 \pm 0.202$ & $1.26 \pm 0.187$ & 0.050 \\
\hline ApoB (g/L) & $0.93 \pm 0.24$ & $0.85 \pm 0.25$ & $0.84 \pm 0.24$ & $0.73 \pm 0.157$ & $<0.0001$ \\
\hline HDL-Ch (mmol/L) & $1.05(0.83-1.25)$ & $1.00(0.84-1.17)$ & $1.05(0.88-1.21)$ & $1.15(0.98-1.32)$ & 0.005 \\
\hline LDL-Ch (mmol/L) & $2.52(1.83-2.95)$ & $2.30(1.71-2.92)$ & $2.28(1.60-2.84)$ & $2.14(1.72-2.76)$ & 0.240 \\
\hline $\mathrm{Lp}(\mathrm{a})(\mathrm{mg} / \mathrm{L})$ & $199.40(97.00-477.33)$ & $137.45(70.40-332.25)$ & $134.10(71.10-429.00)$ & $118(55.2-183)$ & 0.001 \\
\hline TCh (mmol/L) & $4.53(3.79-5.18)$ & $4.21(3.57-4.90)$ & $4.08(3.43-4.87)$ & $3.83(3.39-4.39)$ & $<0.0001$ \\
\hline $\mathrm{TG}(\mathrm{mmol} / \mathrm{L})$ & $1.41(1.07-2.23)$ & $1.25(0.99-1.78)$ & $1.32(0.90-1.68)$ & $0.97(0.75-1.18)$ & $<0.0001$ \\
\hline sdLDL (mmol/L) & $0.92(0.58-1.22)$ & $0.78(0.54-1.06)$ & $0.71(0.48-1.01)$ & $0.57(0.46-0.75)$ & $<0.0001$ \\
\hline $\mathrm{UA}(\mu \mathrm{mol} / \mathrm{L})$ & $372.50(322.50-447.75)$ & $362.50(299.50-432.25)$ & $386.00(307.00-486.00)$ & $259(210-296)$ & $<0.0001$ \\
\hline Urea (mmol/L) & $4.77(3.92-5.61)$ & $5.31(4.25-6.43)$ & $5.22(4.30-6.44)$ & $4.56(3.85-5.65)$ & 0.002 \\
\hline $\mathrm{Cr}(\mu \mathrm{mol} / \mathrm{L})$ & $66.50(55.50-77.00)$ & $73.00(63.00-84.50)$ & $72.00(58.00-89.00)$ & $55(49-62)$ & $<0.0001$ \\
\hline
\end{tabular}

$\varepsilon 2$ allele $=\varepsilon 2 / \varepsilon 2+\varepsilon 2 / \varepsilon 3$ genotypes; $\varepsilon 3$ allele $=\varepsilon 3 / \varepsilon 3+\varepsilon 2 / \varepsilon 4$; genotype; $\varepsilon 4$ allele $=\varepsilon 3 / \varepsilon 4+\varepsilon 4 / \varepsilon 4$ genotypes. All values were adjusted for ethnicity, age and gender except the PWV was adjusted for age, SBP and ethnicity. Biological reference interval: ApoA1: 1-1.6 g/L; ApoB: 0.75-1.0 g/L; HDL-Ch: 1.0-1.55 mmol/L; LDL-Ch: 1.9-3.1 mmol/L; Lp(a): <300 mg/L; TCh: 3.1-5.2 mmol/L; TG: 0.56-1.70 mmol/L; sdLDL: 0.25-1.17

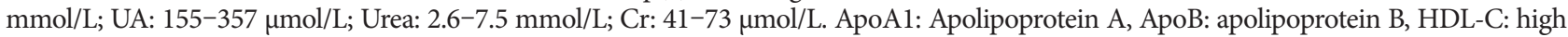
density lipoprotein cholesterol, LDL-C: low density lipoprotein cholesterol, Lp(a): lipoprotein (a), TCh: total cholesterol, TG: triglyceride, sdLDL: small dense density lipoprotein, UA: uric acid, Cr: creatinine, WML: White matter lesions 
brain atrophy group $(\mathrm{p}>0.05)$. The same conclusion appeared in the combined metabolic disease group ( $\mathrm{p}>0.05)$.

\section{Comparison of metabolic parameters among the different $A p o E$ alleles}

We finally analyzed the effects of the $A P O E$ alleles on the

Table 2. Association of apoE Allele and genotype between disease group and control group

\begin{tabular}{|c|c|c|c|c|}
\hline Clinical characteristics & WML & Brain atrophy & Co-occurrence of WML and brain atrophy & $\mathrm{p}$ value \\
\hline Genotype (\%) & & & & 0.016 \\
\hline$E 2 / 2$ & $2(3.45)$ & $0(0.00)$ & $2(1.79)$ & \\
\hline$E 2 / 3$ & $3(5.17)$ & $13(10.16)$ & $25(22.32)$ & \\
\hline$E 2 / 4$ & $0(0.00)$ & $3(2.34)$ & $2(1.79)$ & \\
\hline$E 3 / 3$ & $38(65.52)$ & $89(69.53)$ & $64(57.14)$ & \\
\hline$E 3 / 4$ & $13(22.41)$ & $23(17.97)$ & $17(15.18)$ & \\
\hline$E 4 / 4$ & $2(3.45)$ & $0(0.00)$ & $2(1.79)$ & \\
\hline Allele (\%) & & & & 0.011 \\
\hline E2 & $5(8.62)$ & $13(10.16)$ & $27(24.11)$ & \\
\hline E3 & $38(65.52)$ & $92(71.88)$ & $66(58.93)$ & \\
\hline E4 & $15(25.86)$ & $23(17.97)$ & $19(16.96)$ & \\
\hline \multicolumn{5}{|c|}{ Combined metabolic disease (\%) } \\
\hline Hyperuricemia & $16(27.59)$ & $30(23.44)$ & $37(33.04)$ & 0.254 \\
\hline Hypertension & $42(72.41)$ & $79(61.72)$ & $75(66.96)$ & 0.343 \\
\hline Hyperhomocysteinemia & $12(20.69)$ & $46(35.94)$ & $28(25.00)$ & 0.054 \\
\hline Hyperlipidemia & $20(34.48)$ & $33(25.78)$ & $32(28.57)$ & 0.477 \\
\hline Type 2 diabetes & $11(18.97)$ & $43(33.59)$ & $21(18.75)$ & 0.015 \\
\hline
\end{tabular}

$\varepsilon 2$ allele $=\varepsilon 2 / \varepsilon 2+\varepsilon 2 / \varepsilon 3$ genotypes; $\varepsilon 3$ allele $=\varepsilon 3 / \varepsilon 3$ genotype; $\varepsilon 4$ allele $=\varepsilon 3 / \varepsilon 4+\varepsilon 4 / \varepsilon 4$ genotypes. WML: White matter lesions

Table 3. Comparison of baseline characteristics of participants with $E 2$, E3, and E4 alleles in WML

\begin{tabular}{|c|c|c|c|c|}
\hline Genotype & $\mathrm{E} 2(\mathrm{~N}=5)$ & $\mathrm{E} 3(\mathrm{~N}=38)$ & $\mathrm{E} 4(\mathrm{~N}=15)$ & $\mathrm{p}$ value \\
\hline Gender (male\%) & $4(4 / 5)$ & $15(15 / 38)$ & $10(10 / 15)$ & 0.076 \\
\hline Age (years) & $60.40 \pm 5.550$ & $61.79 \pm 10.624$ & $66.53 \pm 9.234$ & 0.026 \\
\hline ApoA1 (g/L) & $1.50 \pm 0.255$ & $1.32 \pm .234$ & $1.36 \pm 0.162$ & 0.218 \\
\hline ApoB (g/L) & $1.05(1.04-1.24)$ & $0.85(0.77-1.03)$ & $0.89(0.69-1.02)$ & 0.040 \\
\hline HDL-Ch (mmol/L) & $1.23 \pm 0.430$ & $1.07 \pm 0.342$ & $1.05 \pm 0.202$ & 0.516 \\
\hline LDL-Ch (mmol/L) & $3.02 \pm 1.085$ & $2.49 \pm 0.821$ & $2.38 \pm 0.816$ & 0.342 \\
\hline $\mathrm{Lp}(\mathrm{a})(\mathrm{mg} / \mathrm{L})$ & $208.00(92.30-1000.10)$ & $190.30(85.25-343.13)$ & $222.50(100-727)$ & 0.717 \\
\hline $\mathrm{TCh}(\mathrm{mmol} / \mathrm{L})$ & $5.15 \pm 1.020$ & $4.56 \pm 1.076$ & $4.49 \pm 0.944$ & 0.453 \\
\hline TG $(\mathrm{mmol} / \mathrm{L})$ & $1.28(1.04-2.46)$ & $1.36(0.97-2.23)$ & $1.52(1.08-2.41)$ & 0.895 \\
\hline sdLDL (mmol/L) & $1.08 \pm 0.254$ & $0.94 \pm 0.381$ & $0.87 \pm 0.423$ & 0.569 \\
\hline $\mathrm{UA}(\mu \mathrm{mol} / \mathrm{L})$ & $331(239-377.5)$ & $398(335.5-456.5)$ & $381(297-444)$ & 0.102 \\
\hline Urea $(\mathrm{mmol} / \mathrm{L})$ & $5.12(4.72-6.62)$ & $5.13(4.20-5.99)$ & $3.83(3.21-4.78)$ & 0.021 \\
\hline $\mathrm{Cr}(\mu \mathrm{mol} / \mathrm{L})$ & $63(47-84.5)$ & $66.5(56.75-73.25)$ & $69(51-88)$ & 0.937 \\
\hline \multicolumn{5}{|c|}{ Combined metabolic disease (\%) } \\
\hline Hyperuricemia & $1(20.00)$ & $10(26.35)$ & $5(33.33)$ & 0.809 \\
\hline Hypertension & $5(100)$ & $24(63.15)$ & $13(86.67)$ & 0.080 \\
\hline Hyperhomocysteinemia & $3(60.00)$ & $6(15.79)$ & $3(20.00)$ & 0.072 \\
\hline Hyperlipidemia & $2(13.33)$ & $12(31.58)$ & $6(40.00)$ & 0.814 \\
\hline Type 2 diabetes & $0(0.00)$ & $8(21.05)$ & $3(20.00)$ & 0.525 \\
\hline
\end{tabular}

$\varepsilon 2$ allele $=\varepsilon 2 / \varepsilon 2+\varepsilon 2 / \varepsilon 3$ genotypes; $\varepsilon 3$ allele $=\varepsilon 3 / \varepsilon 3+\varepsilon 2 / \varepsilon 4$, genotype; $\varepsilon 4$ allele $=\varepsilon 3 / \varepsilon 4+\varepsilon 4 / \varepsilon 4$ genotypes. WML: White matter lesions 
Table 4. Comparison of baseline characteristics of participants with $E 2, E 3$ and $E 4$ alleles in brain atrophy

\begin{tabular}{|c|c|c|c|c|}
\hline Genotype & $\mathrm{E} 2(\mathrm{~N}=13)$ & $E 3(\mathrm{~N}=92)$ & $\mathrm{E} 4(\mathrm{~N}=23)$ & $\mathrm{p}$ value \\
\hline Gender (male\%) & $11(11 / 13)$ & $67(67 / 92)$ & $14(14 / 23)$ & 0.292 \\
\hline Age (years) & $69(63.5-76)$ & $68(6325-78.75)$ & $74(68-83)$ & 0.054 \\
\hline ApoA1 (g/L) & $1.33 \pm 0.193$ & $1.32 \pm 0.210$ & $1.26 \pm 0.193$ & 0.423 \\
\hline $\operatorname{ApoB}(g / L)$ & $0.88 \pm 0.327$ & $0.86 \pm 0.236$ & $0.80 \pm 0.241$ & 0.599 \\
\hline $\mathrm{HDL}-\mathrm{Ch}(\mathrm{mmol} / \mathrm{L})$ & $1(0.88-1.26)$ & $1.01(0.84-1.13)$ & $0.98(0.85-1.24)$ & 0.874 \\
\hline LDL-Ch (mmol/L) & $2.8(1.48-3.27)$ & $2.3(1.72-2.96)$ & $2.25(1.76-2.75)$ & 0.839 \\
\hline $\mathrm{Lp}(\mathrm{a})(\mathrm{mg} / \mathrm{L})$ & $254(54.95-461)$ & $131.5(70.4-317.75)$ & $137(73.7-345)$ & 0.831 \\
\hline $\mathrm{TCh}(\mathrm{mmol} / \mathrm{L})$ & $4.06(3.37-5.16)$ & $4.28(3.67-4.97)$ & $3.85(3.24-4.37)$ & 0.376 \\
\hline $\mathrm{TG}(\mathrm{mmol} / \mathrm{L})$ & $1.24(1.04-2.51)$ & $1.27(0.98-1.83)$ & $1.17(0.89-1.50)$ & 0.518 \\
\hline sdLDL (mmol/L) & $0.89 \pm 0.374$ & $0.83 \pm 0.389$ & $0.78 \pm 0.440$ & 0.763 \\
\hline $\mathrm{UA}(\mu \mathrm{mol} / \mathrm{L})$ & $381.62 \pm 89.511$ & $369.64 \pm 116.971$ & $346.74 \pm 94.852$ & 0.595 \\
\hline Urea $(\mathrm{mmol} / \mathrm{L})$ & $4.82(3.4-5.88)$ & $5.34(4.35-6.45)$ & $5.77(4.04-6.44)$ & 0.277 \\
\hline $\mathrm{Cr}(\mu \mathrm{mol} / \mathrm{L})$ & $76(66-80.5)$ & $72.5(63.00-85.00)$ & $73.00(62.00-86.00)$ & 0.974 \\
\hline \multicolumn{5}{|c|}{ Combined metabolic disease (\%) } \\
\hline Hyperuricemia & $4(30.77)$ & $23(25.00)$ & $3(13.04)$ & 0.387 \\
\hline Hypertension & $9(69.23)$ & $59(64.13)$ & $11(47.83)$ & 0.299 \\
\hline Hyperhomocysteinemia & $4(30.77)$ & $32(34.78)$ & $10(43.48)$ & 0.680 \\
\hline Hyperlipidemia & $6(46.15)$ & $24(26.09)$ & $3(13.04)$ & 0.092 \\
\hline Type 2 diabetes & $3(23.08)$ & $35(38.04)$ & $5(21.74)$ & 0.233 \\
\hline
\end{tabular}

$\varepsilon 2$ allele $=\varepsilon 2 / \varepsilon 2+\varepsilon 2 / \varepsilon 3$ genotypes; $\varepsilon 3$ allele $=\varepsilon 3 / \varepsilon 3+\varepsilon 2 / \varepsilon 4$, genotype; $\varepsilon 4$ allele $=\varepsilon 3 / \varepsilon 4+\varepsilon 4 / \varepsilon 4$ genotypes

clinical and metabolic parameters in Combined with white matter lesions and brain atrophy group. As shown in Table 5, the levels of ApoB, LDL-Ch, TCh, and sdLDL were significantly different among the APOE genotype groups in Combined with white matter lesions and brain atrophy group. Specifically, the subjects with an alleles E2 was associated with the lower level of ApoB, LDL-Ch, TCh, and sdLDL compared with the E3 and E4 group ( $<<0.0001, \mathrm{p}<0.0001, \mathrm{p}=0.005, \mathrm{p}=$ 0.002 , respectively). In addition, we compared the relationship between ApoE alleles and combined metabolic disease, only Type 2 diabetes of combined metabolic disease was different among different $A P O E$ alleles ( $\mathrm{p}=0.016)$.

\section{Comparison of metabolic parameters among the different disease groups of the subjects with $E 3 / E 3$ genotype}

As shown in Table 6, clinical information including Gender and Age and the levels of sdLDL were significantly different among the different disease groups of the subjects with E3/E3 genotype. Specifically, the subjects with White matter lesions has higher level of sdLDL but lower age and female frequency compared other disease groups $(\mathrm{p}=0.039, \mathrm{p}<0.0001, \mathrm{p}=0.002$, respectively). In addition, we compared the relationship between disease groups of the subjects with E3/E3 genotype and combined metabolic disease, only type 2 diabetes of combined metabolic disease was significantly different in the three disease groups of the subjects with E3/E3 genotype ( $\mathrm{p}=0.004)$.

\section{Comparison of metabolic parameters among the different disease groups of the subjects with E3/E4 genotype}

As shown in Table 7, clinical information including age and the levels of ApoB and TG were significantly different among the different disease groups of the subjects with E3/E4 genotype. Specifically, the subjects with White matter lesions has higher level of TG than other disease groups $(\mathrm{p}=0.013)$, but lower age than other disease groups $(\mathrm{p}=0.001, \mathrm{p}=0.026$, respectively). Interestingly, the subjects with brain atrophy has lower level of ApoB than other disease groups ( $p=0.026$ ). In addition, we compared the relationship between disease groups of the subjects with E3/E4 genotype and combined metabolic disease, there was no significantly different in the three disease groups of the subjects with E3/E4 genotype ( $\mathrm{p}>0.05$ ).

\section{The multivariate logistic regression analysis of Basic Information, Clinical indicators and $A P O E$ among different disease groups \\ Table 8 presents that Gender $(\mathrm{p}=0.012, \mathrm{OR}=3.192,95 \% \mathrm{CI}$ : $1.289-7.904)$ and Age $(\mathrm{p}<0.0001, \mathrm{OR}=0.900,95 \%$ CI: $0.860-$ $0.942)$ of basic information and E2 Allele $(\mathrm{p}=0.019, \mathrm{OR}=0.197$,}


Table 5. Comparison of baseline characteristics of participants with E2, E3, and E4 alleles in co-occurrence of WML and brain atrophy

\begin{tabular}{|c|c|c|c|c|}
\hline Genotype & $\mathrm{E} 2(\mathrm{~N}=27)$ & $\mathrm{E} 3(\mathrm{~N}=66)$ & $\mathrm{E} 4(\mathrm{~N}=19)$ & $\mathrm{p}$ value \\
\hline Gender (male\%) & $18(18 / 27)$ & $41(41 / 66)$ & $14(14 / 19)$ & 0.636 \\
\hline Age (years) & $74(67-80.25)$ & $71(66-78.25)$ & $68(64-77)$ & 0.644 \\
\hline ApoA1 (g/L) & $1.27 \pm 0.184$ & $1.30 \pm 0.218$ & $1.32 \pm 0.169$ & 0.716 \\
\hline $\operatorname{ApoB}(g / L)$ & $0.70 \pm 0.242$ & $0.86 \pm 0.216$ & $0.98 \pm 0.219$ & $<0.0001$ \\
\hline $\mathrm{HDL}-\mathrm{Ch}(\mathrm{mmol} / \mathrm{L})$ & $1.02(0.82-1.19)$ & $1.07(0.86-1.23)$ & $1.06(0.92-1.14)$ & 0.571 \\
\hline LDL-Ch (mmol/L) & $1.75 \pm 0.801$ & $2.31 \pm 0.788$ & $2.74 \pm 0.804$ & $<0.0001$ \\
\hline $\operatorname{Lp}(\mathrm{a})(\mathrm{mg} / \mathrm{L})$ & $131.45(67.82-491)$ & $140.05(79.50-431)$ & $108(54.60-353.10)$ & 0.653 \\
\hline $\mathrm{TCh}(\mathrm{mmol} / \mathrm{L})$ & $3.73 \pm 1.007$ & $4.21 \pm 1.030$ & $4.77 \pm 1.128$ & 0.005 \\
\hline $\mathrm{TG}(\mathrm{mmol} / \mathrm{L})$ & $1.24(0.87-1.68)$ & $1.32(0.90-1.66)$ & $1.46(1.10-2.35)$ & 0.277 \\
\hline sdLDL (mmol/L) & $0.63 \pm .351$ & $0.75 \pm 0.319$ & $1.00 \pm 0.392$ & 0.002 \\
\hline $\mathrm{UA}(\mu \mathrm{mol} / \mathrm{L})$ & $406(294.25-493.75)$ & $360.50(297.25-446.25)$ & $427(326-468)$ & 0.384 \\
\hline Urea $(\mathrm{mmol} / \mathrm{L})$ & $4.79(4.38-6.26)$ & $5.60(4.38-6.63)$ & $4.60(3.90-6.95)$ & 0.348 \\
\hline $\mathrm{Cr}(\mu \mathrm{mol} / \mathrm{L})$ & $76(61-85.50)$ & $68.50(57.00-95.00)$ & $77.00(57.00-93.00)$ & 0.949 \\
\hline \multicolumn{5}{|c|}{ Combined metabolic disease (\%) } \\
\hline Hyperuricemia & $11(40.74)$ & $20(30.30)$ & $6(31.58)$ & 0.617 \\
\hline Hypertension & $19(70.37)$ & $43(65.15)$ & $13(68.42)$ & 0.879 \\
\hline Hyperhomocysteinemia & $6(22.22)$ & 19 (28.79) & $3(15.79)$ & 0.478 \\
\hline Hyperlipidemia & $8(29.63)$ & $18(27.27)$ & $6(46.15)$ & 0.926 \\
\hline Type 2 diabetes & $4(14.81)$ & $9(13.64)$ & $8(42.11)$ & 0.016 \\
\hline
\end{tabular}

$\varepsilon 2$ allele $=\varepsilon 2 / \varepsilon 2+\varepsilon 2 / \varepsilon 3$ genotypes; $\varepsilon 3$ allele $=\varepsilon 3 / \varepsilon 3+\varepsilon 2 / \varepsilon 4$, genotype; $\varepsilon 4$ allele $=\varepsilon 3 / \varepsilon 4+\varepsilon 4 / \varepsilon 4$ genotypes. WML: White matter lesions

Table 6. Comparison of clinical data and complications among three disease groups of APOE E3/E3

\begin{tabular}{|c|c|c|c|c|}
\hline Genotype & WML $(\mathrm{N}=38)$ & Brain atrophy $(\mathrm{N}=89)$ & $\begin{array}{l}\text { Co-occurrence of WML } \\
\text { and brain atrophy }(\mathrm{N}=64)\end{array}$ & $\mathrm{p}$ value \\
\hline Gender (male, \%) & $15(39.47)$ & $65(73.03)$ & $40(62.50)$ & 0.002 \\
\hline Age (years) & $61.79 \pm 10.624$ & $69.61 \pm 9.822$ & $71.78 \pm 8.682$ & $<0.0001$ \\
\hline $\operatorname{ApoA1}(\mathrm{g} / \mathrm{L})$ & $1.32 \pm 0.234$ & $1.32 \pm 0.213$ & $1.30 \pm 0.216$ & 0.738 \\
\hline $\operatorname{ApoB}(g / L)$ & $0.85(0.77-1.03)$ & $0.86(0.64-1.03)$ & $0.87(0.71-1.01)$ & 0.599 \\
\hline HDL-Ch (mmol/L) & $1.02(0.79-1.25)$ & $1.01(0.84-1.13)$ & $1.05(0.86-1.22)$ & 0.852 \\
\hline LDL-Ch (mmol/L) & $2.52(1.83-2.85)$ & $2.30(1.71-2.96)$ & $2.29(1.70-2.88)$ & 0.678 \\
\hline $\mathrm{Lp}(\mathrm{a})(\mathrm{mg} / \mathrm{L})$ & $190.30(85.25-343.13)$ & $132.00(70.15-329.50)$ & $133.55(78.50-423.43)$ & 0.420 \\
\hline $\mathrm{TCh}(\mathrm{mmol} / \mathrm{L})$ & $4.56 \pm 1.076$ & $4.30 \pm 1.020$ & $4.19 \pm 0.986$ & 0.199 \\
\hline $\mathrm{TG}(\mathrm{mmol} / \mathrm{L})$ & $1.36(0.97-2.23)$ & $1.27(0.98-1.82)$ & $1.32(0.91-1.67)$ & 0.287 \\
\hline $\mathrm{sdLDL}(\mathrm{mmol} / \mathrm{L})$ & $0.94 \pm 0.381$ & $0.83 \pm 0.395$ & $0.75 \pm 0.313$ & 0.039 \\
\hline $\mathrm{UA}(\mu \mathrm{mol} / \mathrm{L})$ & $398.00(335.50-456.50)$ & $364.00(302.50-441.00)$ & $363.50(300.25-468.75)$ & 0.312 \\
\hline Urea $(\mathrm{mmol} / \mathrm{L})$ & $5.13(4.20-5.99)$ & $5.36(4.40-6.45)$ & $5.55(4.35-6.68)$ & 0.483 \\
\hline $\mathrm{Cr}(\mu \mathrm{mol} / \mathrm{L})$ & $66.50(56.75-73.25)$ & $72.00(63.00-84.00)$ & $68.50(57.00-95.00)$ & 0.150 \\
\hline \multicolumn{5}{|c|}{ Combined metabolic disease (\%) } \\
\hline Hyperuricemia & $10(26.32)$ & $22(24.72)$ & $20(31.25)$ & 0.663 \\
\hline Hypertension & $24(63.16)$ & $57(64.04)$ & $41(64.06)$ & 0.995 \\
\hline Hyperhomocysteinemia & $6(15.79)$ & $31(34.83)$ & $18(28.13)$ & 0.094 \\
\hline Hyperlipidemia & $12(31.58)$ & $24(26.97)$ & $17(26.56)$ & 0.839 \\
\hline Type 2 diabetes & $8(21.05)$ & $33(37.08)$ & $9(14.06)$ & 0.004 \\
\hline
\end{tabular}

WML: White matter lesions 
Table 7. Comparison of clinical data and complications among three disease groups of APOE E3/E4

\begin{tabular}{|c|c|c|c|c|}
\hline Genotype & WML $(\mathrm{N}=13)$ & brain atrophy $(\mathrm{N}=23)$ & $\begin{array}{l}\text { Co-occurrence of WML } \\
\text { and brain atrophy }(\mathrm{N}=17)\end{array}$ & $\mathrm{p}$ value \\
\hline Gender (male, \%) & $10(76.92)$ & $14(60.87)$ & $13(76.47)$ & 0.462 \\
\hline Age (years) & $63.92 \pm 6.383$ & $74.83 \pm 8.516$ & $71.00 \pm 8.208$ & 0.001 \\
\hline ApoA1 (g/L) & $1.39 \pm .150$ & $1.26 \pm .193$ & $1.33 \pm 0.173$ & 0.095 \\
\hline ApoB (g/L) & $0.93 \pm .220$ & $0.80 \pm .241$ & $1.01 \pm 0.215$ & 0.026 \\
\hline HDL-Ch(mmol/L) & $1.12(0.86-1.23)$ & $098(0.85-1.24)$ & $1.07(0.93-1.29)$ & 0.501 \\
\hline $\mathrm{LDL}-\mathrm{Ch}(\mathrm{mmol} / \mathrm{L})$ & $2.51 \pm 0.783$ & $2.25 \pm 0.765$ & $2.84 \pm 0.796$ & 0.073 \\
\hline $\mathrm{Lp}(\mathrm{a})(\mathrm{mg} / \mathrm{L})$ & $191(89.9-679.1)$ & $137(73.7-345)$ & $108(54.8-301.05)$ & 0.453 \\
\hline $\mathrm{TCh}(\mathrm{mmol} / \mathrm{L})$ & $4.64(3.86-5.13)$ & $3.85(3.24-4.37)$ & $4.76(4.25-5.48)$ & 0.049 \\
\hline $\mathrm{TG}(\mathrm{mmol} / \mathrm{L})$ & $1.53(1.31-2.50)$ & $1.17(0.89-1.50)$ & $1.46(1.13-2.52)$ & 0.013 \\
\hline sdLDL (mmol/L) & $0.96 \pm 0.387$ & $0.78 \pm 0.440$ & $1.03 \pm 0.407$ & 0.174 \\
\hline $\mathrm{UA}(\mu \mathrm{mol} / \mathrm{L})$ & $381.38 \pm 92.151$ & $346.74 \pm 94.852$ & $416.65 \pm 103.780$ & 0.089 \\
\hline Urea $(\mathrm{mmol} / \mathrm{L})$ & $4.24 \pm 1.208$ & $5.43 \pm 1.435$ & $5.16 \pm 1.690$ & 0.071 \\
\hline $\mathrm{Cr}(\mu \mathrm{mol} / \mathrm{L})$ & $66.31 \pm 24.243$ & $74.35 \pm 15.865$ & $73.12 \pm 18.415$ & 0.459 \\
\hline \multicolumn{5}{|l|}{ Combined metabolic disease } \\
\hline Hyperuricemia & $4(30.77)$ & $3(13.04)$ & $6(35.29)$ & 0.226 \\
\hline Hypertension & $11(84.62)$ & $11(47.83)$ & $12(70.59)$ & 0.069 \\
\hline Hyperhomocysteinemia & $3(23.08)$ & $10(43.48)$ & $3(17.65)$ & 0.173 \\
\hline Hyperlipidemia & $5(38.46)$ & $3(13.04)$ & $6(35.29)$ & 0.151 \\
\hline Type 2 diabetes & $2(15.38)$ & $5(21.74)$ & $7(41.18)$ & 0.226 \\
\hline
\end{tabular}

WML: White matter lesions

95\% CI: 0.051-0.762) in White matter lesions group were also significantly associated with disease progression when Combined with white matter lesions and brain atrophy was set as the reference group. Similarly, the results showed that Hyperhomocysteinemia of Combined metabolic disease $(\mathrm{p}=0.023$, $\mathrm{OR}=0.472,95 \% \mathrm{CI}: 0.247-0.900)$ and $\mathrm{UA}(\mathrm{p}=0.004, \mathrm{OR}=0.995$, 95\% CI: 0.992-0.999) in brain atrophy group were also significantly associated with disease progression when Combined with white matter lesions and brain atrophy was set as the reference group.

\section{DISCUSSION}

Some articles have confirmed the clinical results of the presence of WML and brain atrophy on MRI. ${ }^{10}$ Previous studies have found that age and gender are risk factors for brain atrophy and white matter. ${ }^{18,19}$ However, few articles had reported the relationship between gender and alleles, genotypes in different diseases. Here we show that the prevalence of men in brain atrophy, co-occurrence of WML and brain atrophy disease group is higher than that of WML, the co-occurrence of WML and brain atrophy as a control group, the multivariate logistic analysis of clinical data showed that Gender was a risk factor and gender was a protective factor in the WML group in brain atrophy group. More importantly, E4 allele carriers are older than E2 and E3 allele carriers in WML not in other disease groups. We also analyzed the relationship between clinical data, and different disease groups in E3/E3 and E3/E4 genotype group, respectively. The results showed that age, gender were significantly different in different disease groups of E3/E3 carriers, while age was also significantly different in different disease groups of E3/E4 carriers. Interestingly, the age of the cooccurrence group was higher than the other groups in both $E 3 / E 3$ and E3/E4 groups. The above data indicates that age is closely related to the progression and type of disease and genotype, Allele.

According to a previous report cardiovascular disease is associated with high blood lipid levels, type 2 diabetes and other metabolic diseases, such as hyperhomocysteinemia, hyperuricemia, hypertension. ${ }^{20-22}$ In the present study, the metabolic index of the disease group (including WML, brain atrophy, cooccurrence of WML and brain atrophy) was higher than that of the healthy group, although most of the indicators were within the normal reference range. It is worth mentioning that the uric acid concentration in the disease group is higher than the normal range. The multivariate logistic analysis of metabolic indicators showed that serum uric acid was a protective factor in brain atrophy group. 
Table 8. Multivariate logistic regression analysis among different disease groups

\begin{tabular}{|c|c|c|c|c|c|c|}
\hline & \multicolumn{3}{|c|}{ WML } & \multicolumn{3}{|c|}{ Brain atrophy } \\
\hline & OR & 95\% confidence interval & $\mathrm{p}$ value & OR & $95 \%$ confidence interval & $\mathrm{p}$ value \\
\hline \multicolumn{7}{|l|}{ Basic information } \\
\hline Gender & 3.192 & $1.289-7.904$ & 0.012 & 0.707 & $0.353-1.412$ & 0.326 \\
\hline Age & 0.900 & $0.860-0.942$ & $<0.0001$ & 0.995 & $0.963-1.029$ & 0.771 \\
\hline \multicolumn{7}{|l|}{ Combined metabolic disease } \\
\hline Hyperhomocysteinemia & 0.823 & $0.327-2.073$ & 0.680 & 0.472 & $0.247-0.900$ & 0.023 \\
\hline Hyperuricemia & 1.019 & $0.398-2.609$ & 0.968 & 0.843 & $0.400-1.777$ & 0.653 \\
\hline Hypertension & 0.705 & $0.302-1.646$ & 0.419 & 1.291 & $0.705-2.364$ & 0.408 \\
\hline Hyperlipidemia & 1.188 & $0.468-3.015$ & 0.717 & 1.280 & $0.612-2.675$ & 0.512 \\
\hline Diabetes & 1.223 & $0.480-3.114$ & 0.673 & 0.521 & $0.266-1.024$ & 0.059 \\
\hline \multicolumn{7}{|l|}{ Clinical indicators } \\
\hline UA & 0.999 & $0.995-1.003$ & 0.587 & 0.995 & $0.992-0.999$ & 0.004 \\
\hline ApoA1 & 0.562 & $0.021-15.256$ & 0.732 & 1.165 & $0.097-14.003$ & 0.904 \\
\hline ApoB & 0.405 & $0.003-56.254$ & 0.719 & 0.186 & $0.004-8.247$ & 0.385 \\
\hline HDL-Ch & 4.131 & $0.232-73.637$ & 0.335 & 1.861 & $0.220-15.764$ & 0.569 \\
\hline LDL-Ch & 1.755 & $0.351-8.774$ & 0.494 & 2.308 & $0.591-9.014$ & 0.229 \\
\hline $\operatorname{Lp}(\mathrm{a})$ & 1.001 & $0.999-1.002$ & 0.341 & 1.000 & $0.999-1.001$ & 0.427 \\
\hline TCh & 0.652 & $0.172-2.468$ & 0.529 & 0.658 & $0.211-2.049$ & 0.470 \\
\hline TG & 1.437 & $0.649-3.181$ & 0.371 & 1.571 & $0.811-3.043$ & 0.180 \\
\hline sdLDL & 3.007 & $0.337-26.842$ & 0.324 & 1.683 & $0.324-8.739$ & 0.536 \\
\hline Urea & 0.921 & $0.733-1.158$ & 0.480 & 0.963 & $0.810-1.145$ & 0.670 \\
\hline $\mathrm{Cr}$ & 0.997 & $0.975-1.020$ & 0.820 & 1.001 & $0.993-1.009$ & 0.813 \\
\hline \multicolumn{7}{|l|}{ Allele } \\
\hline $\mathrm{E}=2$ & 0.197 & $0.051-0.762$ & 0.019 & 0.514 & $0.191-1.381$ & 0.187 \\
\hline$E=3$ & 0.508 & $0.199-1.296$ & 0.157 & 1.305 & $0.617-2.762$ & 0.486 \\
\hline $\mathrm{E}=4$ & \multicolumn{3}{|c|}{ The reference group } & & & \\
\hline \multicolumn{7}{|l|}{ Genotype } \\
\hline $\mathrm{APOE}=\mathrm{E} 2 / 2+\mathrm{E} 2 / 4+\mathrm{E} 4 / 4$ & 0.527 & $0.082-3.399$ & 0.500 & 0.689 & $0.120-3.964$ & 0.676 \\
\hline $\mathrm{APOE}=\mathrm{E} 2 / 3$ & 0.210 & $0.043-1.026$ & 0.054 & 0.468 & $0.166-1.316$ & 0.150 \\
\hline $\mathrm{APOE}=\mathrm{E} 3 / 3$ & 0.985 & $0.377-2.572$ & 0.976 & 1.154 & $0.531-2.510$ & 0.717 \\
\hline $\mathrm{APOE}=\mathrm{E} 3 / 4$ & \multicolumn{3}{|c|}{ The reference group } & & & \\
\hline
\end{tabular}

co-occurrence of WML and brain atrophy was set as the reference group. WML: White matter lesions

In the Chinese population, the predominant genotype is E3/ $E 3$ in the Chinese population, whereas the most common allele is E3, followed by E2/E3, E3/E4, E2/E4, E4/E4, and E2/E2. ${ }^{23-25}$ Our research also confirmed that E3/E3 and E3 were dominant. However, in this research, the E3/E4 genotype follows the $E 3 / E 3$ genotype in WML group and brain atrophy group, respectively, but the E2/E2 genotype follows the E2/E3 genotype in the co-occurrence of WML and brain atrophy disease group. The same conclusion is given for E2 and E4 alleles. The difference in the above results may be that the proportion of $A P O E$ genotypes is related to the type of disease.

Since $A P O E$ gene polymorphism plays a key role in the reg- ulation of plasma lipid levels, ${ }^{26,27}$ APOE isoforms are thought to play an important role in the pathogenesis of vascular disease. ${ }^{28,29}$ The results show that, First, serum ApoB and urea levels of patients with WML are different among allele groups. In addition, ApoB level of E2 allele carriers was higher than others. Second, there is no difference in clinical data and metabolic indicators between different alleles of brain atrophy patients. Then, ApoB, LDL-Ch, TCh and sdLDL levels of the metabolic indicators of co-occurrence of WML and brain atrophy patients are significantly different among different alleles. It is worth mentioning that ApoB, LDL-Ch, TCh and sdLDL levels of E2 carriers was lower than that of E3 and E4 
carriers. The above conclusions can be speculated that the relationship between alleles and metabolic indicators is closely related to disease type and progression. Interestingly, genotype has a certain relationship with disease progression. Further analysis of genotypes and metabolic indicators revealed that sdLDL was significantly different in different disease groups of E3/E3 carriers, while APOB and TG were significantly different in different disease groups of E3/E4 carriers.

Metabolic disorder can lead to a greater potential of type 2 diabetes, lipid disorders, cardiovascular disease, hepatic steatosis, and other circulatory disorders. ${ }^{30,31}$ We found that the incidence of type 2 diabetes has significant differences among three disease groups. In addition, type 2 diabetes also has significant differences among different allele groups of co-occurrence group, not WML and brain atrophy groups. Further comparison of the incidence of metabolic complications among different disease groups of the certain genotype, and found that type 2 diabetes has significant differences among different diseases groups of E3/E3 carriers, rather than E3/E4 carriers.

At last, multivariate logistic regression analysis of the factors affecting the disease progression, the co-infected group as a control group, the data show that gender is a risk factor [3.192 (1.289-7.904)], age [0.900 (0.860-0.942)] and E2 allele [0.197 (0.051-0.762)] are protective factors for white matter disease. In the same way, hyperhomocysteinemia [0.472 (0.247$0.900)]$ and uric acid [0.995 $(0.992-0.999)]$ are protective factors for brain atrophy.

\section{Acknowledgments}

None.

\section{Conflicts of Interest}

The authors have no potential conflicts of interest to disclose.

\section{Author Contributions}

Conceptualization: ZhiLi Niu. Data curation: ZhiLi Niu. Formal analysis: PingAn Zhang. Funding acquisition: Dong Li. Investigation: ChengLiang Zhu. Methodology: LiNa Feng. Project administration: Ge Xiong. Resources: NaNa Song. Software: Pei Tang. Supervision: PingAn Zhang. Validation: Feng Liu. Visualization: ZhiLi Niu. Writing_original draft: ZhiLi Niu. Writing—review \& editing: ZhiLi Niu.

\section{ORCID iDs}

$\begin{array}{ll}\text { ZhiLi Niu } & \text { https://orcid.org/0000-0002-3667-1338 } \\ \text { PingAn Zhang } & \text { https://orcid.org/0000-0001-6429-6742 } \\ \text { Dong Li } & \text { https://orcid.org/0000-0002-8728-3271 } \\ \text { ChengLiang Zhu } & \text { https://orcid.org/0000-0002-1120-4418 } \\ \text { LiNa Feng } & \text { https://orcid.org/0000-0002-9638-9916 } \\ \text { Ge Xiong } & \text { https://orcid.org/0000-0002-8849-2809 } \\ \text { NaNa Song } & \text { https://orcid.org/0000-0001-5994-627X } \\ \text { Pei Tang } & \text { https://orcid.org/0000-0002-4294-6350 } \\ \text { Feng Liu } & \text { https://orcid.org/0000-0002-8582-6711 }\end{array}$

\section{REFERENCES}

1. Das HK, McPherson J, Bruns GA, Karathanasis SK, Breslow JL. Isola- tion, characterization, and mapping to chromosome 19 of the human apolipoprotein E gene. J Biol Chem 1985;260:6240-6247.

2. Svobodova H, Kucera F, Stulc T, Vrablík M, Amartuvshin B, Altannavch Ts, et al. Apolipoprotein E gene polymorphism in the Mongolian population. Folia Biol (Praha) 2007;53:138-142.

3. Yousuf FA, Iqbal MP. Review: Apolipoprotein E (Apo E) gene polymorphism and coronary heart disease in Asian populations. Pak J Pharm Sci 2015;28:1439-1444.

4. Kotaska K, Kolarova J, Kotrcova K, Cepova J, Prusa R. Correlation between common genetic variants and risk factors associated with prediction of cardiovascular diseases in dyslipidemic patients. Genet Test Mol Biomarkers 2012;16:210-214.

5. Muller M, Appelman AP, van der Graaf Y, Vincken KL, Mali WP, Geerlings MI. Brain atrophy and cognition: interaction with cerebrovascular pathology? Neurobiol Aging 2011;32:885-893.

6. Resnick SM, Pham DL, Kraut MA, Zonderman AB, Davatzikos C. Longitudinal magnetic resonance imaging studies of older adults: a shrinking brain. J Neurosci 2003;23:3295-3301.

7. Hachinski VC, Potter P, Merskey H. Leuko-araiosis: an ancient term for a new problem. Can J Neurol Sci 1986;13:533-534.

8. Raji CA, Lopez OL, Kuller LH, Carmichael OT, Longstreth WT Jr, Gach HM, et al. White matter lesions and brain gray matter volume in cognitively normal elders. Neurobiol Aging 2012;33:834.e7-e16.

9. Schmidt R, Grazer A, Enzinger C, Ropele S, Homayoon N, PlutaFuerst A, et al. MRI-detected white matter lesions: do they really matter? J Neural Transm (Vienna) 2011;118:673-681.

10. Appelman AP, Exalto LG, van der Graaf Y, Biessels GJ, Mali WP, Geerlings MI. White matter lesions and brain atrophy: more than shared risk factors? A systematic review. Cerebrovasc Dis 2009;28:227-242.

11. Callisaya ML, Beare R, Moran C, Phan T, Wang W, Srikanth VK. Type 2 diabetes mellitus, brain atrophy and cognitive decline in older people: a longitudinal study. Diabetologia 2019;62:448-458.

12. Richter N, Michel A, Onur OA, Kracht L, Dietlein M, Tittgemeyer M, et al. White matter lesions and the cholinergic deficit in aging and mild cognitive impairment. Neurobiol Aging 2017;53:27-35.

13. Alaylioglu M, Gezen-Ak D, Dursun E, Bilgiç B, Hanağası H, Ertan T, et al. The association between clusterin and APOE polymorphisms and late-onset alzheimer disease in a Turkish cohort. J Geriatr Psychiatry Neurol 2016;29:221-226.

14. Araki S, Moczulski DK, Hanna L, Scott LJ, Warram JH, Krolewski AS. APOE polymorphisms and the development of diabetic nephropathy in type 1 diabetes: results of case-control and family-based studies. Diabetes 2000;49:2190-2195.

15. Rudilosso S, San Roman L, Blasco J, Hernandez-Perez M, Urra X, Chamorro A. Evaluation of white matter hypodensities on computed tomography in stroke patients using the Fazekas score. Clin Imaging 2017;46:24-27.

16. Jeong EO, Choi SW, Lim JW, Kwon HJ, Kim SH, Koh HS, et al. Effectiveness of Cortical Atrophy Scale and Indirect Indices of Brain Atrophy to Predict Chronic Subdural Hematoma in Older Patients. Korean J Neurotrauma 2016;12:112-117.

17. Reymer PW, Groenemeyer BE, van de Burg R, Kastelein JJ. Apolipoprotein E genotyping on agarose gels. Clin Chem 1995;41:1046-1047.

18. Aribisala BS, Valdes Hernandez MC, Royle NA, Morris Z, Muñoz Maniega S, Bastin ME, et al. Brain atrophy associations with white matter lesions in the ageing brain: the Lothian Birth Cohort 1936. Eur Radiol 2013;23:1084-1092.

19. Schwichtenberg J, Al-Zghloul M, Kerl HU, Wenz H, Hausner L, Frölich $\mathrm{L}$, et al. Late-onset major depression is associated with age-related white matter lesions in the brainstem. Int J Geriatr Psychiatry 2017;32: 446-454.

20. Conte MS, Desai TA, Wu B, Schaller M, Werlin E. Pro-resolving lipid mediators in vascular disease. J Clin Invest 2018;128:3727-3735.

21. Sattar N. Advances in the clinical management of type 2 diabetes: a brief history of the past 15 years and challenges for the future. BMC 
Med 2019;17:46.

22. Wassink AM, Van Der Graaf Y, Soedamah-Muthu SS, Spiering W, Visseren F. Metabolic syndrome and incidence of type 2 diabetes in patients with manifest vascular disease. Diab Vasc Dis Res 2008;5:114122.

23. Han S, Xu Y, Gao M, Wang Y, Wang J, Liu Y, et al. Serum apolipoprotein $\mathrm{E}$ concentration and polymorphism influence serum lipid levels in Chinese Shandong Han population. Medicine (Baltimore) 2016;95: e5639.

24. Liu H, Mao P, Xie C, Xie W, Wang M, Jiang H. Apolipoprotein E polymorphism and the risk of intracranial aneurysms in a Chinese population. BMC Neurol 2016;16:14.

25. Zhong Z, Wu H, Ye M, Yang Y, Luo W, Wu Y, et al. Association of APOE Gene Polymorphisms with Cerebral Infarction in the Chinese Population. Med Sci Monit 2018;24:1171-1177.

26. Bea AM, Lamiquiz-Moneo I, Marco-Benedi V, Mateo-Gallego R, Pérez-Calahorra S, Jarauta E, et al. Lipid-lowering response in subjects with the p.(Leu167del) mutation in the APOE gene. Atherosclerosis
2019;282:143-147.

27. Liu L, Hu Q, Wu H, Wang X, Gao C, Chen G, et al. Dietary DHA/EPA ratio changes fatty acid composition and attenuates diet-induced accumulation of lipid in the liver of ApoE(-/-) mice. Oxid Med Cell Longev 2018;2018:6256802.

28. Larifla L, Armand C, Bangou J, Blanchet-Deverly A, Numeric P, Fonteau $\mathrm{C}$, et al. Association of APOE gene polymorphism with lipid profile and coronary artery disease in Afro-Caribbeans. PLoS One 2017; 12:e0181620.

29. Raffeld MR, Biffi A, Battey TW, Ayres AM, Viswanathan A, Greenberg $\mathrm{SM}$, et al. APOE epsilon4 and lipid levels affect risk of recurrent nonlobar intracerebral hemorrhage. Neurology 2015;85:349-356.

30. Mendrick DL, Diehl AM, Topor LS, Dietert RR, Will Y, La Merrill MA, et al. Metabolic syndrome and associated diseases: from the bench to the clinic. Toxicol Sci 2018;162:36-42.

31. Vikram NK. Cardiovascular and metabolic complications- diagnosis and management in obese children. Indian J Pediatr 2018;85:535-545. 